\title{
Application of PMU on Fault Detection and Hybrid State Estimations
}

\author{
Andrew Lockie, Thomas Dowd
}

\begin{abstract}
It is feasible to record and monitor the power systems' dynamic behavior with the extensive deployment of phasor measurement units (PMU). PMU provides high-precision data in which has shown applications in many fields. This study gives a comprehensive prospective of the application of $P M U$ in fault detection. Basically, this paper evaluates the application of this data for fault detection. In which, it is crucial to detect the faults at their early stage so remedial reaction can be taken to reduce the potential damage. Second, the incorporation of the PMU data into state estimation algorithms to achieve more stable and accurate estimation algorithm. It is demonstrated, incorporating the PMU data will increase the observability of the system, however more comprehensive algorithm to monitor the system and detect the faults is needed.
\end{abstract}

Keywords: Fault detection, Hybrid state estimation, PMU data, Power systems, SCADA.

\section{INTRODUCTION}

It is important to detect and isolate the faults in their early stage for reliable operation of power systems. It is also crucial to monitor the state of the system. With the extensive deployment of phasor measurement unit (PMU) devices, tracking and observing the power systems' performance has become feasible. Basically, PMU provides time-synchronized measurements of voltage and currents with a GPS synchronized data that are sampled at rate of either 30 or 60 samples per second. Because PMU is well synchronized and provide high-precision data, these data has shown auspicious application in many fields such as power system monitoring, state estimation, power system protection, power system control, simulation validation and fault detection and location. In this report, we will study the application of PMU in fault detection and location impose systems. It has been reported that every early GPS-based PMU units were invented in early 1980s.

Precise State estimation plays imperative role to security and reliability of power systems. State Estimation (SE) methods are used to increase the safety and efficacy of power systems [1]. Historically, SE algorithms trusted solely on SCADA to record and track the systems. Phasor

Revised Manuscript Received on July 02, 2020.

* Correspondence Author

Andrew Lockie*, Department of Physics, Marquette University, Milwaukee, USA.

Thomas Dowd, Department of Physics, Marquette University, Milwaukee, USA.

(C) The Authors. Published by Blue Eyes Intelligence Engineering and Sciences Publication (BEIESP). This is an open access article under the CC BY-NC-ND license (http://creativecommons.org/licenses/by-nc-nd/4.0/) measurement data is extensively incorporated and influence in State Eastmain algorithms after massive deployment of them in the power systems. Recently, it is shown that integrating PMU data would increase the accuracy of the methods that estimate the systems' state. Therefore, a clear picture of most recent hybrid state estimation methods and their application is evaluated in this study.

\section{FAULT DETECTION}

O operate a power system reliably, it is imperative to detect and isolate power system faults in their early stage. With the extensive deployment of phasor measurement units (PMU), it brought many benefits to us and it made it easy to observe the systems' dynamic behavior. Voltage and current measurements are measured and delivered by PMU in a synchronized and time stamped manner. The sampling rate is usually between 30 to 60 samples per seconds. Because PMU is well synchronized and provide high-precision data, these data has shown auspicious application in many fields such as power system monitoring, state estimation, power system protection, power system control, simulation validation and fault detection and location. In this report, we will study the application of PMU in fault detection and location impose systems. It has been reported that the every early GPS-based PMU units were invented in early 1980s [44]. To detect and locate the fault using PMU measurements, Jiang et al. in [1], [2] proposed an on-line method that can deal effectively with numerous distinction features related to precision of fault locators. Locating the fault in 2 steps: pre-location step and correction step, a PMU based method is introduced in [3] to locate the fault for series compensated lines. The advantage of this method is that it does not uses model and knowledge of the operation mode of series devices. Using synchronized PMU data and considering arc voltage discrimination, Lin et al. in[4], [5] proposed an algorithm to detect and locate the fault that are permanent in high voltage power lines. To increase the accuracy of fault isolation, an allocation algorithm is proposed in [6] that determines optimal sets of PMU units. Using circuit analysis based procedure, Brahma in [7] developed a multi-terminal algorithm that compares pre-fault and post-fault synchronized voltage measurements to locate the precise location of the fault. Using the PMU measurements at two-terminals of the transmission lines and arc voltage amplitude, Lee et al. [8], [9] proposed an algorithm to locate the fault in power systems and distinguish the permanent fault from transient fault.

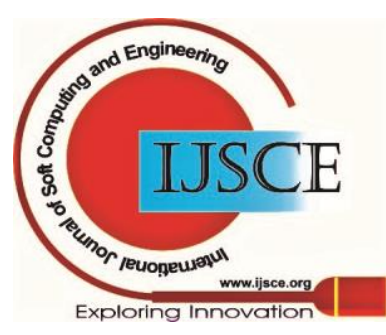




\section{Application of PMU on Fault Detection and Hybrid State Estimations}

To decrease the transmission delay in an adaptive communication network of PMUs and monitoring center, Chuang et al. [10] introduced a computer based algorithm and integrated it with a fault location algorithm.

To estimate the location of a fault in a three-terminal system, Izykowski et al. in [11] used voltage and current that are installed at different locations.

To detect system single line outage, Tate et al. [12] utilized system topology information and real time PMU measurements. This algorithm is further developed in [13] for double line outages detection.

To avoid the negative impact of current transformers in distance protection, Firouzjah et al in [14]proposed a method to identify the fault location in two or three-terminal transmission lines using voltage measurements.

To locate the single to ground faults, Esmaeilian et al in [15] introduced an algorithm that utilizes PMU data in three terminal transmission lines.

Because provided data by electric utility is not reflect the environmental effect, Al-Mohammed in [16] [17]propose a method that uses only PMU data to increase the fault location accuracy.

\section{HYBRID STATE ESTIMATION}

State Estimation (SE) is considered as a vital means for the safe and cost-effective operation of power systems [18] [22]. Traditionally, SE has only utilized SCADA data to observe and document the events in the system. After arrival and advancement of measurement units, engineers and research scholars started to take advantage of its unit features and use it for state estimation algorithms to increase the implementation and precision. Comparing to that come from SCADA, PMUs deliver more harmonized and precise measurements at a higher rate.

However, there are two major issues to integrate PMU data in SE algorithms which are: 1) both SCADA and PMU measurements are recorded at different rate [19], and 2) PMU measurements and tradition SE algorithms are not compatible [19]. In addition, State estimation with only SCADA has low sampling rate (one sample for every 5 seconds) which decrease the visibility of the systems and it also reduces the response rates to any change. State estimation only PMU data has observability issues. The system is not fully observable due to limited number of installed PMUs at buses. The advantage of hybrid state estimation is that it updates the states with PMU and SCADA data which results in increase observability of the system. Generally, the past research that proposed using PMU and SCADA for state estimation can be categorized into the ones that only use PMU data and the ones that use both data also known as Hybrid State Estimation (HSE).

Authors in [20] studied an algorithm which used predictor-corrector IP (PCIP) approach for state estimation. This algorithm has many type of Flexible alternating current transmission systems (FACTS) devices in different formats.

To simplify the weighted least squares (WLS) SE method, authors of [21] uses the complex Taylor series expansion. It is important to mention that this method is built on Wirtinger calculus. It is not a difficult task to apply the WLS state estimator in complex variables. However, the real Gauss-Newton methods is capable of effortlessly using complex measurements at the same time holding the high accuracy.

Where the problem is PMU placement, authors in [23] proposed a systematic optimal multistage PMU placement method for enhancing hybrid state estimation.

Authors in [24] studied an SE algorithm to deal with the non-Gaussian noise and skewness. To establish the suitable length and weights of measurements, Mahalanbis distances approach was integrated into a statistical hypothesis test. Generalized maximum-likelihood (SHGM)-estimator utilized the weights to filter out non-Gaussian noise.

This paper [27] studies features affecting state estimation precision. These features are uncertainties in measurement, number measurements. This study also introduces pseudo power measurements.

Authors in [28] proposed a hybrid state estimation (HSE) to integrate PMU data into established weighted least square state estimators. PMU data are translated into the SCADA data when we only have PMU measurement and it utilized the date in Weighted Least Square-based state estimator. The algorithm of proposed method is demonstrated in Fig. 1. Proposed optimization function is also described in (1).

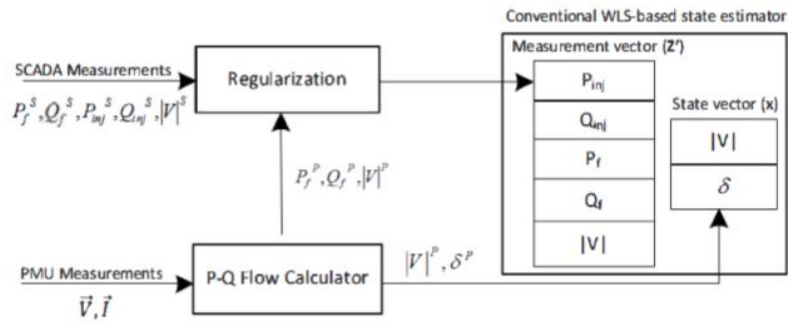

Fig. 1.Algorithm of proposed method in [27].

$$
\begin{aligned}
& \min _{\boldsymbol{x}} J(\boldsymbol{x})=[\boldsymbol{z}-\boldsymbol{h}(\boldsymbol{x})]^{T} \boldsymbol{R}^{-1}[\mathbf{z}-\boldsymbol{h}(\boldsymbol{x})] \\
& \boldsymbol{Z}=\left[\boldsymbol{P}_{i n j}^{S}, \boldsymbol{Q}_{i n j}^{S}, \boldsymbol{P}_{f}^{S}, \boldsymbol{Q}_{f}^{S},|\boldsymbol{V}|^{S}\right]^{T} \\
& \boldsymbol{z}^{\prime}=\left[\boldsymbol{P}_{i n j}^{S}, \boldsymbol{Q}_{i n j}^{S}, \boldsymbol{P}_{f}^{S}, \boldsymbol{Q}_{f}^{S}, \boldsymbol{P}_{f}^{P}, \boldsymbol{Q}_{f}^{P},|\boldsymbol{V}|^{S},|\boldsymbol{V}|^{P}\right]^{T}
\end{aligned}
$$

Regarding this method and its optimization function, it is important to note:

1- Used White Gaussian Noise

2- State vector contains only $|\mathbf{V}|, \delta$

3 -The setback is an uncertain number of equations when just PMU measurements are available due to insufficient number of PMUs. This could be addressed through regularization which is basically using the distributed Compressive Sensing [42].

To advance the a more traditional averaging window algorithm for state estimation, authors of [29] proposed a method which relies on signal to establish the length of window. In other words, the quantity of measurements that are used to approximate the underlying average of PMU measurements, which are noisy, is calculated using a method that is depended on signal. It uses the Shewhart method for detecting the changes for used

to observing the

Published By:

Blue Eyes Intelligence Engineering

and Sciences Publication

(C) Copyright: All rights reserved.

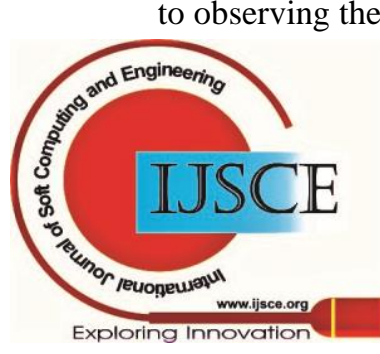


changes and identify the measurements that are buffered integrating pseudo-measurements obtained by the originatin g PMU bus branches.

Authors in [31] proposed to use an optimization algorithm which relies on particle swarm that are hybrid for state estimation(SE).

Using convergence, observability and performance as enhancing hybrid state estimation.

A direct hybrid constrained state estimator (HCSE) is proposed in [33]. In order to avoid uncertainties, this authors used the untransformed data that comes from PMU.

To enhance calculation performance of hybrid state estimators, a decentralization method that utilizes sensitivity proposed in [34]. The algorithm of proposed method is illustrated in figure 2.

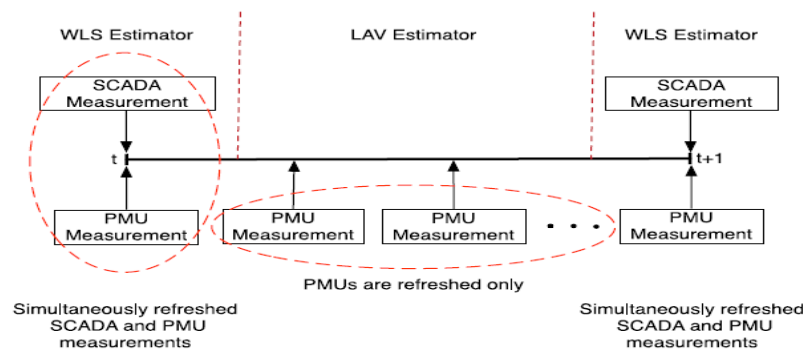

Fig. 2.Algorithm of method disscussed in [34].

The authors in [38] studied a PMU-based HSE that uses the correlation between time and space to deliver refusal of bad data and advance the security and precision. Steps taken to implement this algorithm is illustrated in figure 3. It is also important to note that authors suggested to use buffering method to overcome the issue with difference in the rates between SCADA data and PMU data. This permit utilizing phasor measurement in the proposed hybrid estimator algorithm.

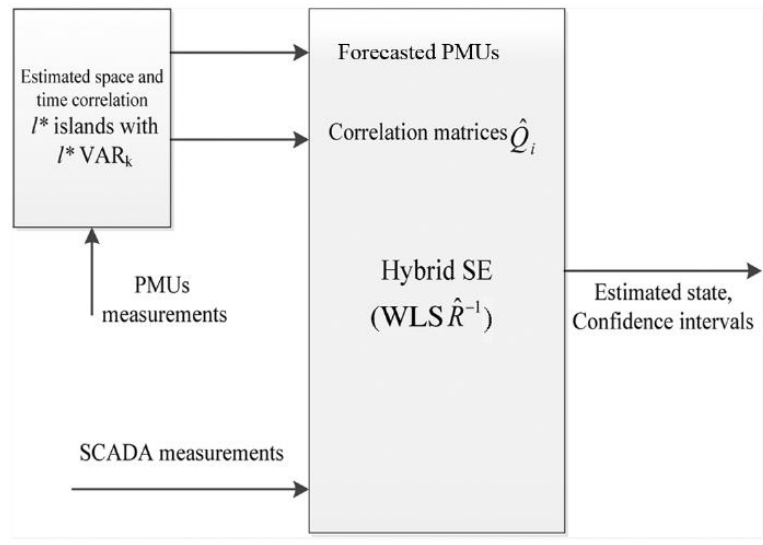

Fig. 3.Algorithm of proposed method in [38].

To estimate Jacobian matrix, authors in [40] offered a hybrid method that uses both PMU and SCADA data. This algorithm is model based. This method is shown in details in figure 4 . [30]. Authors in [29] studied a restricted formulation of HSE metrics, PMU placement problem is studied in [32] for matrix that relates measurement errors to state estimates is

Step 1: Estimate $\delta$ and $\omega$ from PMU measurements.

Step 2: Calculate the sample covariance matrix $Q_{\delta \delta}$ and $Q_{\omega \omega}$ by (15)-(16).

Step 3: Estimate the Jacobian matrix $\frac{\partial \boldsymbol{P}_{\boldsymbol{e}}}{\partial \boldsymbol{\delta}}$ by (17).

Step 4: If the damping $D$ of generators are known, construct the system state matrix:

$$
A=\left[\begin{array}{cc}
0 & I_{n} \\
-M^{-1} \frac{\partial \boldsymbol{P}_{\boldsymbol{e}}}{\partial \boldsymbol{\delta}} & -M^{-1} D
\end{array}\right]
$$

Fig. 4.Algorithm of proposed method in [40].

In [41], combined a PMU placement method and another PMU observability method to find the best place for PMU placement.

To continuously regulate the weight of measurements with respect to the distance of disturbances, a PMU-based state estimation method (adaptive weight assignment function) is proposed in [43].

\section{RESUTLS ANALYSIS}

This section we analyze the aforementioned studies.

While studying the proposed algorithm in [34], it is important to consider following points:

1- WLS is computationally effective algorithm. However, it is very venerable to bad data and it can significantly affect its performance.

2- Least absolute value (LAV) is a computationally expensive method when SCADA and PMU exist at the same time and it has to calculate for both data set.

3- This algorithm utilizes minimum required set of old SCADA to maintain observability [35] [36].

It is crucial to point a few comments with respect to the proposed algorithm in [40]:

1- It makes the assumption that at every substation there is at least one PMU installed which is not happening at least in near future

2- Rotor speed and angle is calculated using PMU data for oscillations.

3- The generator mode that is used in this study is classical model which is mainly approved by researchers and its performance is relatively acceptable [37]-[39].

Beside all the aforementioned methods for state estimation and fault detection, several imminent gaps exist that needs to be studied such as:

(1) The relation between length of data and speed of fault detection, as PMU sampling rate increases the fault detection can be done faster.

Published By:

Blue Eyes Intelligence Engineering

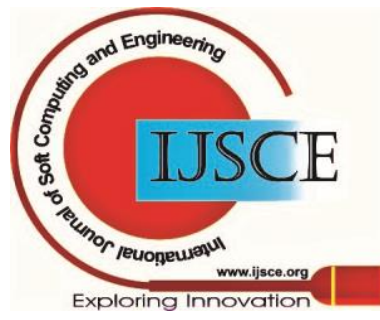




\section{Application of PMU on Fault Detection and Hybrid State Estimations} state estimation is more accurate than conventional algorithms because of high sampling rate in PMU data.

Someone should answer this question that can we find a method that utilizes only PMU data and has the same accuracy as if using hybrid method.

(1) Using estimation of PMU data at the locations that PMUs are not installed instead of using SCADA data.

\section{CONCLUSION}

This study mainly discussed PMU application in power systems for fault detection and HSE. Basically, it investigates two application of PMU data. First it evaluates the application of this data for fault detection. As it is discussed throughout this paper, it is crucial to detect the faults at their early stage so remedial reaction can be taken to reduce the potential damage. Second, this paper investigates the incorporation of the PMU data into state estimation algorithms to achieve more stable and accurate estimation algorithm. As it has shown, incorporating the PMU data will increase the observability of the system. However, there is a need for a comprehensive algorithm to monitor the system and detect the faults. Although PMU data has improved many algorithms such as state estimations, it has not been used to its potential.

\section{REFERENCES}

1. J. Jiang, J. Yang, Y. Lin, C. Liu, and J. Ma, “An Adaptive PMU Based Fault Detection / Location Technique for Transmission Lines Part I: Theory and Algorithms," IEEE Transantions Power Deliv., vol. 15, no. 2, pp. 486-493, 2000.

2. J. A. Jiang, Y. H. Lin, J. Z. Yang, T. M. Too, and C. W. Liu, “An adaptive PMU based fault detection/location technique for transmission lines - Part II: PMU implementation and performance evaluation," IEEE Trans. Power Deliv., vol. 15, no. 4, pp. 1136-1146, 2000.

3. C. S. Yu, C. W. Liu, S. L. Yu, and J. A. Jiang, "A new PMU-based fault location algorithm for series compensated lines," IEEE Trans. Power

4. M. Ghorbaniparvar, N. Zhou, and X. Li. "Coherence function estimation with a derivative constraint for power grid oscillation detection." In 2016 IEEE Global Conference on Signal and Information Processing (GlobalSIP), pp. 791-795. IEEE, 2016.

5. Y. H. Lin, C. W. Liu, and C. S. Chen, "A new PMU-based fault detection/location technique for transmission lines with consideration of arcing fault discrimination - Part II: Performance Evaluation," IEEE Trans. Power Deliv., vol. 19, no. 4, pp. 1587-1593, 2004

6. R. a. F. Pereira, L. G. W. Da Silva, and J. R. S. Mantovani, "PMUs optimized allocation using a tabu search algorithm for fault location in electric power distribution system," 2004 IEEE/PES Transm. Distrib. Conf. Expo. Lat. Am. (IEEE Cat. No. 04EX956), pp. 143-148, 2004

7. S. M. Brahma, "Fault location scheme for a multi-terminal transmission line using synchronized voltage measurements," IEEE Trans. Power Deliv., vol. 20, no. 2 II, pp. 1325-1331, 2005.

8. C. J. Lee, J. B. Park, J. R. Shin, and Z. M. Radojevi??, "A new two-terminal numerical algorithm for fault location, distance protection, and arcing fault recognition," IEEE Trans. Power Syst., vol. 21, no. 3,

9. C. L. Ó, J. Park, J. Shin, and Z. Radojevi, “Two Terminals Numerical Algorithm for Distance Protection, Fault Location and Acing Faults Recognition Based on Synchronized Phasors," J. Electr. Eng. Technol., vol. 1, no. 1, pp. 35-41, 2006.

10. C. L. Chuang, J. A. Jiang, Y. C. Wang, C. P. Chen, and Y. T. Hsiao, "An adaptive PMU-based fault location estimation system with a fault-tolerance and load-balancing communication network," 2007 IEEE Lausanne POWERTECH, Proc., pp. 1197-1202, 2007.

11. J. Izykowski, E. Rosolowski, M. M. Saha, M. Fulczyk, and P. Balcerek, "A fault-location method for application with current differential relays of three-terminal lines," IEEE Trans. Power Deliv., vol. 22, no. 4, pp. 2099-2107, 2007.

12. J. E. Tate and T. J. Overbye, "Line Outage Detection Using Phasor Angle Measurements," vol. 23, no. 4, pp. 1644-1652, 2008.
(1) Based on many studies, it has been suggested that hybrid Deliv., vol. 17, no. 1, pp. 33-46, 2002. pp. 1460-1462, 2006.

13. J. E. Tate and T. J. Overbye, "Double Line outage detection using phasor angle measurements," IEEE Trans. Power Syst., vol. 23, no. 4, pp. 1644-1652, 2009.

14. K. G. Firouzjah and A. Sheikholeslami, "A current independent method based on synchronized voltage measurement for fault location on transmission lines," Simul. Model. Pract. Theory, vol. 17, no. 4, pp. 692-707, 2009.

15. a. Esmaeilian, M. Mohseninezhad, M. Doostizadeh, and M. Khanabadi, "A precise PMU based fault location method for multi terminal transmission line using voltage and current measurement," 2011 10th Int. Conf. Environ. Electr. Eng., pp. 1-4, 2011.

16. M. Ghorbaniparvar, N. Zhou, X. Li, Daniel J. Trudnowski, and Ruichao Xie. "A forecasting-residual spectrum analysis method for distinguishing forced and natural oscillations." IEEE Transactions on Smart Grid 10, no. 1 (2017): 493-502.

17. A. H. Al-Mohammed and M. A. Abido, "A fully adaptive PMU-based fault location algorithm for series-compensated lines," IEEE Trans. Power Syst., vol. 29, no. 5, pp. 2129-2137, 2014.

18. A. Abur and A. G. Exposito, Power system state estimation: theory and impLementation, Marcel Dekker Inc, 2004.

19. A. Costa, A. Albuquerque and D. Bez, "An Estimation Fusion Method for Including Phasor Measurements into Power System RealTime Modeling," IEEE Transactions on Power Systems, vol. 28, no. 2, pp. 1910-1920, 2013.

20. C. Rakpenthai, S. Premrudeepreechacharn and S. Uatrongjit, "Power system with multi-type FACTS devices states estimation based on predictor-corrector interior point algorithm," Electrical power and energy system, vol. 31, no. 4, pp. 160-166, 2009.

21. I. Dzafic, R. Jabr and T. Hrnjic, "Hybrid State Estimation in Complex Variables," IEEE Transactions on Power Systems 1, 2018.

22. M. Ghorbaniparvar, "Survey on forced oscillations in power system." Journal of Modern Power Systems and Clean Energy 5, no. 5 (2017): 671-682.

23. J. Hazra, K. Das, B. Roy, M. Padmanaban and A. Sinha, "Multistage Optimal PMU Placement for Hybrid State Estimaiton," in In Power \& Energy Society General Meeting, 2017.

24. J. Zhou and L. Mili, "A Framework for Robust Hybrid State Estimation with Unknown Measurement Noise Statistics," IEEE Transactions on Industrial Informatics, 2017.

25. M. Jabbar, and H. Ghorbaniparvar. "Use of GC-MS combined with resolution methods to characterize and to compare the essential oil components of green and bleached cardamom." IJRCE 5 (2014).

26. X. Yu, H. Zhang and J. Cao, "Analysis ofInfluencing Factors in Hybrid State Estimation," in IEEE PES Asia-Pacific Power and Energy Conference, Xi'an, China, 2016.

27. R. Hamidi, H. Khodabandelou, H. Livani and M. Sami-Fadali, "Hybrid State Estimation using Distributed Compressive Sensing," in Power and Energy Society General Meeting (PESGM), 2016.

28. N. Kashyap, S. Werner and J. Lunden, "Signal-Dependent Preprocessing of Buffered PMU Measurements for Hybrid State Estimation," in Instrumentation and Measurement Technology Conference Proceedings (I2MTC), 2016 IEEE International, Taipei, Taiwan, 2016.

29. M. Asprou and E. Kyriakides, "Enhancement of hybrid state estimation using pseudo flow measurements," in Power and Energy Society General Meeting, 2011.

30. M. Jabbar, and H. Ghorbaniparvar. "Determination of Volatile Components in Black Cardamom with Gas Chromatography-Mass Spectrometry and Chemometric Resolution ." International Journal of Engineering Research and Technology 3 (11), 1280-1286 2014

31. S. Nanchian, A. Majumdar and B. Pal, "Three-Phase State Estimation Using Hybrid Particle Swarm Optimization," IEEE TRANSACTIONS ON SMART GRID, vol. 8, no. 3, 2017.

32. X. Li, A. Scaglione and T. Chang, "A Framework for Phasor Measurement Placement in Hybrid State Estimation Via Gauss-Newton," IEEE TRANSACTIONS ON POWER SYSTEMS, vol. 29, no. 2, 2014.

33. G. Valverde, S. Chakrabarti, E. Kyriakides and V. Terzija, "Constrained Formulation for Hybrid State Estimation," IEEE TRANSACTIONS ON POWER SYSTEMS,, vol. 26, no. 3, 2011.

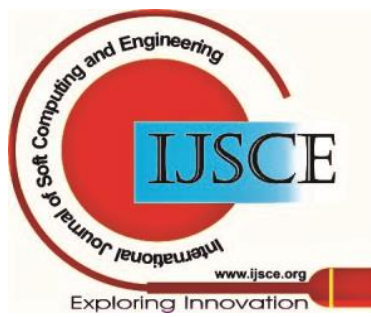


34. M. Gol, "A Decentralization Method for Hybrid State Estimators," IEEE TRANSACTIONS ON POWER SYSTEMS, vol. 33, no. 2, 2018.

35. Ghorbaniparvar, Mohammadreza, Xiaohua Li, and Ning Zhou. "Demand side management with a human behavior model for energy cost optimization in smart grids." In Signal and Information Processing (GlobalSIP), 2015 IEEE Global Conference on, pp. 503-507. IEEE, 2015.

36. M. Jabbar, and E. Konoz. "The combination of GC-MS with MCR Method to characterize and to compare the essential oil components of two types of cardamom" 20th Iranian Analytical Chemistry Conference

37. M. Jabbar, H. Ghorbaniparvar, F. Ghorbaniparvar, "Sensitivity of the General Linear Model to Assumptions" International Journal of Innovative Science and Modern Engineering, 2020

38. Y. Chakhchoukh, V. Vittal, G. Heydt and H. Ishii, "LTS-Based Robust Hybrid SE Integrating Correlation," IEEE TRANSACTIONS ON POWER SYSTEMS, vol. 23, no. 4, 2017.

39. F. Ghorbaniparvar, and H. Sangrody. "PMU application for locating the source of forced oscillations in smart grids." In IEEE Power and Energy Conference at Illinois (PECI), pp. 1-5. IEEE, 2018.

40. X. Wang, J. Bialek and K. Turitsyn, "PMU-Based Estimation of Dynamic State Jacobian Matrix and Dynamic System State Matrix in Ambient Conditions," IEEE TRANSACTIONS ON POWER SYSTEMS, vol. 33, no. 1, 2018.

41. P. Ghosh, "Complete and incomplete PMU observablity for hybrid state estimation," in International Conference on Computation of Power, Energy Information and Commuincation (ICCPEIC), 2017.

42. M. Ghorbaniparvar., and N. Zhou. "Bootstrap-based hypothesis test for detecting sustained oscillations." In Power \& Energy Society General Meeting, 2015 IEEE, pp. 1-5. IEEE, 2015.

43. J. Zhou, G. Zhang, K. Das, G. Korres and N. Manousakis, "Power System Real-Time Monitoring by Using PMU-Based Robust State Estimation Method," IEEE Transaction on Smart Grid, vol. 7, no. 1, 2016.

44. M. Ghorbaniparvar and F. Ghorbaniparvar, "Portfolio optimization applied for wholesale electricity spot market (wesm) based on markowitz theory". International Journal of Science and Modern Engineering,. 2013.

45.A. Sasani, S. Tibado, "Stock Market Prediction using Hidden Markov Model and Neural Network ". International Journal of Engineering and Applied Sciences, 2020 https://dx.doi.org/10.31873/IJEAS.7.04.07

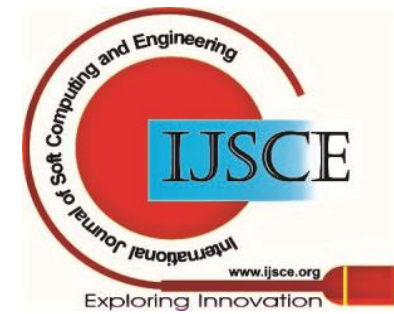

